\title{
Comparação entre a evolução de pré-escolares com fibrose cística identificados por triagem neonatal ou por sintomatologia clínica
}

\author{
Comparison between the outcome of preschool children with cystic fibrosis \\ identified by newborn screening or by clinical symptoms
}

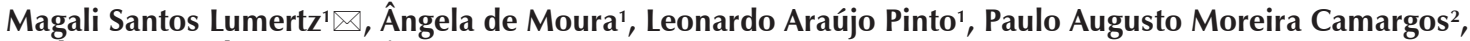 \\ Paulo José Cauduro Marostica ${ }^{3}$ \\ ${ }^{1}$ Serviço de Pneumologia Pediátrica da Pontifícia Universidade Católica do Rio Grande do Sul (PUCRS). Porto Alegre, RS \\ 2 Departamento de Pneumologia Pediátrica da Universidade Federal de Minas Gerais (UFMG). Belo Horizonte, MG \\ 3 Serviço de Pneumologia Pediátrica do Hospital de Clínicas de Porto Alegre (HCPA). Porto Alegre, RS.
}

Como citar este artigo (How to cite this article):

Lumertz MS, de Moura A, Pinto LA, Camargos PAM, Marostica PJC. Comparação entre a evolução de pré-escolares com fibrose cística identificados por triagem neonatal ou por sintomatologia clínica (Comparison between the outcome of preschool children with cystic fibrosis identified by newborn screening or by clinical symptoms). Sci Med. 2018;28(2):ID29566. http://doi.org/10.15448/1980-6108.2018.2.29566

\section{RESUMO}

OBJETIVOS: Comparar dados clínicos, laboratoriais e espirométricos de pacientes pré-escolares (idade entre três e seis anos) com fibrose cística, identificados pela alteração do tripsinogênio imunorreativo no teste de triagem neonatal (grupo IRT) com os dados de pacientes cuja suspeita diagnóstica ocorreu a partir de sinais e sintomas clínicos (grupo DC).

MÉTODOS: Estudo do tipo coorte retrospectiva, com amostra obtida a partir dos prontuários dos pacientes que faziam acompanhamento ambulatorial especializado e multidisciplinar. Todas as crianças incluídas tinham diagnóstico confirmado de fibrose cística por teste de eletrólitos no suor e/ou sequenciamento genético. Como variáveis incluíram-se sexo, peso, altura, índice de massa corporal, número de internações por ano, colonização por Pseudomonas aeruginosa, presença de insuficiência pancreática e escore de Shwachman. Este escore abrange quatro domínios: atividade geral, nutrição, exame radiológico e avaliação física, cada um pontuado entre 5 e 25 pontos, sendo que o escore $\leq 40$ pontos indica estado grave e $\geq 86$ pontos excelente estado. Para comparação da função pulmonar utilizaram-se os dados da última espirometria realizada antes dos seis anos de idade. Os desfechos foram comparados por qui-quadrado ou teste t de Student, com limite de significância de 0,05 .

RESULTADOS: Foram incluídos no estudo 24 pacientes, sete no grupo IRT e 17 no grupo DC. As crianças do grupo IRT iniciaram

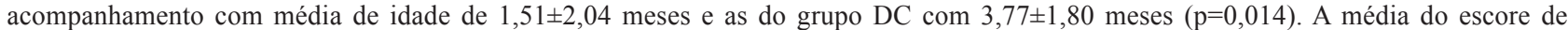
Shwachman foi $94,40 \pm 2,19$ nos pacientes do grupo IRT $v s$. $87,67 \pm 8,00$ no grupo $D C$ ( $p=0,018$ ). A média do escore $Z$ para o índice de massa corporal foi $0,95 \pm 1,02$ no grupo IRT vs. $0,51 \pm 1,05$ no grupo $\mathrm{DC}(\mathrm{p}=0,051)$. Foram encontrados valores ligeiramente superiores nas variáveis espirométricas no grupo IRT, sem diferença estatisticamente significativa entre os grupos.

CONCLUSÕES: Os resultados sugerem que os pacientes diagnosticados com fibrose cística a partir da suspeita pelo teste de triagem neonatal beneficiaram-se de uma intervenção precoce, podendo iniciar os testes de função pulmonar e receber tratamento e orientações mais cedo. A avaliação de parâmetros clínicos com o escore de Shwachman mostrou que os benefícios puderam ser observados já na idade pré-escolar.

DESCRITORES: fibrose cística; espirometria; triagem neonatal; triagem do recém-nascido; nutrição da criança.

\section{ABSTRACT}

AIMS: To compare clinical, laboratory and spirometric parameters of preschoolers (ages from three to six years old) with cystic fibrosis identified by abnormal newborn screening test (NS group), with data of patients whose diagnostic suspicion arose from characteristic clinical signs and symptoms (CS group).

METHODS: Retrospective cohort study, with a sample obtained from the medical charts of patients who received specialized and multidisciplinary outpatient follow-up. All the included children had a confirmed diagnosis of cystic fibrosis by sweat electrolyte testing and or genetic sequencing. Variables included sex, weight, height, body mass index, number of hospitalizations per year, Pseudomonas aeruginosa colonization, presence of pancreatic insufficiency and Shwachman score, which covers four domains: general activity, nutrition, radiological examination and physical evaluation, each one scored between 5 and 25 points. A total score $\leq 40$ points indicates severe state, and $\geq 86$ points indicates excellent state. For comparison of pulmonary function, data from the last spirometry performed before the age of six years were used. Outcomes were compared by chi-square or Student's $t$ test, with a significance limit of 0.05 .

RESULTS: Twenty-four patients were included in the study, seven children in the NS group and 17 children in the CS group. The children of the NS group started follow-up with a mean age of $1.51 \pm 2.04$ months, and those of the CS group started with $3.77 \pm 1.80$ months ( $\mathrm{p}=0.014$ ). The mean Shwachman score was $94.40 \pm 2.19$ in the NS vs. $87.67 \pm 8.00$ in the CS group ( $\mathrm{p}=0.018$ ). The mean $\mathrm{Z}$ score for body mass index was 
$0.95 \pm 1.02$ in the NS group vs. $0.51 \pm 1.05$ in the CS group $(\mathrm{p}=0.051)$. Spirometric variables had slightly higher values in the NS group, with no statistically significant difference between groups.

CONCLUSIONS: The results suggest that those patients diagnosed with cystic fibrosis from the neonatal screening test suspicion benefited from an early intervention, being able to initiate pulmonary function tests and receive treatment and counseling earlier. Evaluation of clinical parameters with the Shwachman score showed that benefits could be already observed at the preschool age.

KEYWORDS: cystic fibrosis; lung function; neonatal screening; infant, newborn, screening; child nutrition.

Abreviaturas: $\mathrm{CVF}$, capacidade vital forçada; DC, diagnóstico após suspeita clínica; $\mathrm{FC}$, fibrose cística; $\mathrm{FEF}_{25-75}$, fluxo expiratório forçado entre $25 \%$ e $75 \%$ da capacidade vital forçada; IMC, índice de massa corporal; IRT, tripsinogênio imunorreativo (immunoreactive trypsinogen); TTN, teste de triagem neonatal; $\mathrm{VEF}_{1}$, volume expiratório forçado no primeiro segundo; $\mathrm{VEF}_{0,5}$, volume expiratório forçado na metade do primeiro segundo; $\mathrm{VEF}_{0,75}$, volume expiratório forçado a $75 \%$ do primeiro segundo.

\section{INTRODUÇÃO}

A fibrose cística (FC), embora seja uma doença com envolvimento de múltiplos órgãos, tem como principal causa de morbimortalidade o acometimento pulmonar. Medidas de função pulmonar, como a espirometria, oferecem dado objetivo do comprometimento pulmonar e permitem o acompanhamento a longo prazo da evolução da doença $[1,2]$.

A suspeita diagnóstica advém da presença de um conjunto de sinais e sintomas clínicos característicos ou de um valor alterado de tripsinogênio imunorreativo (IRT, immunoreactive trypsinogen) quando da realização do teste de triagem neonatal (TTN). Quando ocorre a suspeita pelo TTN, muitos pacientes ainda são assintomáticos, logo, passíveis de intervenções profiláticas e terapêuticas mais precoces e eficazes. A pesquisa do IRT foi incorporada ao TTN em diferentes momentos na rede pública de saúde do Brasil, sendo que no estado do Rio Grande do Sul foi incluído a partir de junho de 2012. Até então, era disponível somente no TTN realizado por laboratórios privados [1,3-5].

Há diversos estudos acerca dos benefícios em longo prazo do TTN para FC em relação ao aspecto nutricional, e alguns acerca da função pulmonar em adolescentes e adultos. Entretanto, existem poucos dados relacionando o impacto do diagnóstico precoce nos desfechos clínicos e funcionais de crianças préescolares [5-9]. O objetivo deste estudo foi comparar os dados clínicos, laboratoriais e espirométricos de pacientes pré-escolares portadores de FC, entre o grupo cuja suspeita diagnóstica foi levantada a partir da pesquisa do IRT no TTN e o grupo de pacientes cuja suspeita ocorreu apenas após o surgimento de sinais e sintomas clínicos característicos.

\section{MÉTODOS}

Foi realizado um estudo de coorte retrospectiva por revisão de prontuários, incluindo pacientes com FC que se encontravam em acompanhamento ambulatorial regular, especializado e multidisciplinar no ambulatório do Centro de Fibrose Cística da Pontifícia Universidade Católica do Rio Grande do Sul (PUCRS), um serviço de nível terciário localizado no Hospital São Lucas da PUCRS, em Porto Alegre. O estudo foi aprovado pelo Comitê de Ética em Pesquisa da universidade (parecer consubstanciado de número 186.516).

Foram revisados dados de prontuários de crianças portadoras de FC com idade entre três e seis anos. As informações foram coletadas utilizando uma ficha padronizada. Foram incluídas as informações dos pacientes cujos registros continham dados de espirometria. Nesse serviço são registrados apenas resultados de espirometrias cujas curvas foram aceitáveis e reprodutíveis, seguindo os critérios da American Thoracic Society (ATS).

Os pacientes foram distribuídos em dois grupos, conforme o critério que originou a suspeita diagnóstica de FC. Aqueles encaminhados ao ambulatório devido a sinais e sintomas clínicos sugestivos (como infecções respiratórias de repetição, baixo ganho ponderal, esteatorreia, entre outros) originaram o grupo DC (diagnóstico após suspeita clínica). Já os pré-escolares cuja suspeita surgiu a partir da alteração do IRT ao TTN formaram o grupo IRT. Todos os pacientes selecionados tinham diagnóstico confirmado de FC por teste de eletrólitos no suor (concentração de cloro superior a $60 \mathrm{mmol} / \mathrm{L}$ ) e/ou por sequenciamento genético.

Como variáveis clínicas e laboratoriais incluíram-se sexo, peso, altura, índice de massa corporal (IMC), número de internações por ano, colonização por Pseudomonas aeruginosa, presença de insuficiência pancreática (definida pela necessidade de uso de 
enzimas pancreáticas pelo paciente), valor do escore de Shwachman [10], valor do cloro ao teste de eletrólitos no suor, e descrição do genótipo daqueles que o possuíam. Foi definida como colonização crônica por $P$. aeruginosa a presença de ao menos $50 \%$ das culturas em um ano com resultado positivo $[11,12]$.

$\mathrm{O}$ escore de Shwachman serve como estimativa da evolução da doença associada à FC. Este abrange quatro domínios: atividade geral, nutrição, exame radiológico e avaliação física. Cada área é pontuada entre 5 e 25 pontos, sendo o escore estratificado e considerado como grave quando a soma é igual ou inferior a 40 pontos e como excelente quando apresenta 86 ou mais pontos [10].

Todos os pacientes incluídos realizaram pelo menos uma espirometria com menos de seis anos de idade, onde se avaliaram os valores absolutos do volume expiratório forçado no primeiro segundo $\left(\mathrm{VEF}_{1}\right)$, da capacidade vital forçada (CVF), da relação entre $\mathrm{VEF}_{1} / \mathrm{CVF}$, e do fluxo expiratório forçado entre $25 \%$ e $75 \%$ da $\mathrm{CVF}\left(\mathrm{FEF}_{25-75}\right)$ e seus percentis, além do escore $\mathrm{Z}$ para idade, altura e sexo em relação à equação internacional [13]. Os exames de espirometria foram realizados com o equipamento Koko (PDS
Instrumentation, Inc., Louisville, CO, EUA). Para comparação da função pulmonar entre os grupos utilizaram-se os dados da última espirometria realizada antes dos seis anos de idade. Todos os procedimentos estavam de acordo com os critérios estabelecidos pela ATS $[14,15]$.

Para análise estatística, as variáveis numéricas foram descritas em média e desvio padrão. As variáveis categóricas foram relatadas em número absoluto e percentual. Os desfechos foram comparados por quiquadrado (para variáveis categóricas) ou teste $t$ de Student (para variáveis contínuas). Aceitou-se limite de significância de 0,05 . O banco de dados foi criado no programa Excel, versão 14.0 e a análise estatística foi realizada no programa SPSS for Windows versão 16.0 .

\section{RESULTADOS}

Foram incluídos 24 pacientes, sendo 13 (54,2\%) do sexo masculino. Em sete crianças a suspeita de FC foi pelo TTN, compondo o grupo IRT, e em 17 crianças o diagnóstico foi feito após o surgimento de sinais e sintomas característicos da FC (Tabela 1).

Tabela 1. Comparação das características de crianças com fibrose cística, entre aquelas cuja suspeita diagnóstica surgiu na triagem neonatal por alteração do tripsinogênio imunorreativo (grupo IRT) e aquelas cuja suspeita diagnóstica ocorreu pelo surgimento de sinais e sintomas clínicos (grupo DC).

\begin{tabular}{|c|c|c|c|}
\hline Características & $\begin{array}{c}\text { Grupo IRT } \\
\mathbf{n}=7\end{array}$ & $\begin{array}{c}\text { Grupo DC } \\
n=17\end{array}$ & $\mathbf{p}$ \\
\hline Sexo masculino - $\mathrm{n}(\%)$ & $03(43 \%)$ & $10(59 \%)$ & 0,476 \\
\hline Idade ao início do acompanhamento (anos) - média \pm DP & $1,51 \pm 2,04$ & $3,77 \pm 1,80$ & 0,014 \\
\hline Idade à realização da 1ạ espirometria (anos) - média „DP & $4,46 \pm 0,74$ & $5,17 \pm 0,58$ & 0,021 \\
\hline Média de idade à realização das espirometrias - média $\pm D P$ & $4,86 \pm 0,87$ & $5,39 \pm 0,48$ & 0,002 \\
\hline Escore de Shwachman - média \pm DP & $94,40 \pm 2,19$ & $87,67 \pm 8,00$ & 0,018 \\
\hline Escore $\mathrm{Z}$ do IMC - média $\pm \mathrm{DP}$ & $0,95 \pm 1,02$ & $0,51 \pm 1,05$ & 0,051 \\
\hline Percentil do IMC - média \pm DP & $73,03 \pm 27,34$ & $63,61 \pm 29,63$ & 0,128 \\
\hline Insuficiência pancreática - n (\%) & $05(71 \%)$ & $16(94 \%)$ & 0,127 \\
\hline Número de internações hospitalares por ano - média \pm DP & $0,29 \pm 0,49$ & $1,00 \pm 1,22$ & 0,160 \\
\hline Valor do cloro ao teste de suor $(\mathrm{mmol} / \mathrm{L})-$ média $\pm \mathrm{DP}$ & $104,67 \pm 57,14$ & $77,88 \pm 15,63$ & 0,100 \\
\hline Infecção crônica por Pseudomonas aeruginosa (n \%) & $02(28,57 \%)$ & $04(23,52 \%)$ & 0,586 \\
\hline Pacientes usuários de convênio privado - n (\%) & $07(100,0 \%)$ & $12(70,58 \%)$ & 0,272 \\
\hline CVF em percentual do previsto - média \pm DP & $107,16 \pm 21,47$ & $94,28 \pm 31,19$ & 0,262 \\
\hline $\mathrm{VEF}_{1}$ em percentual do previsto - média $\pm \mathrm{DP}$ & $102,57 \pm 21,14$ & $92,62 \pm 24,06$ & 0,352 \\
\hline $\mathrm{VEF}_{1} / \mathrm{CVF}$ em percentual do previsto - média $\pm \mathrm{DP}$ & $95,55 \pm 7,02$ & $91,32 \pm 7,49$ & 0,215 \\
\hline $\mathrm{FEF}_{25-75}$ em percentual do previsto - média $\pm \mathrm{DP}$ & $100,54 \pm 34,69$ & $80,12 \pm 31,52$ & 0,175 \\
\hline Número de pacientes com internação no último ano & $02(28 \%)$ & $08(47 \%)$ & 0,653 \\
\hline
\end{tabular}

Grupo IRT, diagnóstico após alteração do tripsinogênio imunorreativo na triagem neonatal; grupo DC, diagnóstico após suspeita clínica; DP, desvio padrão; IMC, índice de massa corporal; $C V F$, capacidade vital forçada; $\mathrm{VEF}_{1}$, volume expiratório forçado no primeiro segundo; $\mathrm{FEF}_{25-75}$, volume expiratório forçado entre $25 \%$ e $75 \%$ da capacidade vital forçada. Teste do qui-quadrado (variáveis categóricas) ou teste t de Student (variáveis contínuas). 
Conforme visualizado na Tabela 1, as crianças do grupo IRT iniciaram acompanhamento ambulatorial com uma média de idade menor do que aquelas do grupo DC. Quatro pacientes do grupo IRT começaram seguimento neste centro multidisciplinar especializado até os quatro meses de idade, tendo os demais iniciado após o primeiro ano de vida (aos 1,3, aos 2,8 e aos 5,6 anos de idade respectivamente) porque vinham em acompanhamento prévio em consultórios privados. A maioria dos pacientes de ambos grupos apresentava insuficiência pancreática e um quarto das crianças era cronicamente colonizada por $P$. aeruginosa (Tabela 1).

Encontrou-se diferença significativa $(p=0,018)$ nos valores médios do escore de Shwachman entre o grupo IRT $(94,40 \pm 2,19)$ e o grupo DC $(87,67 \pm 8,00)$, indicando melhor evolução clínica do grupo IRT (Tabela 1). A idade em que o escore foi calculado foi semelhante entre os dois grupos: $5,40 \pm 0,40$ anos no grupo IRT e 5,42 $\pm 0,49$ anos no grupo $\mathrm{DC}(\mathrm{p}=0,934)$.

Verificou-se tendência de superioridade na média do escore $\mathrm{Z}$ para o IMC nas crianças do grupo IRT $(0,95 \pm 1,02)$ em comparação àquelas do grupo DC $(0,51 \pm 1,05)$ quando da realização das espirometrias, mas com valor limítrofe para significância estatística: $\mathrm{p}=0,051$.

Em relação ao número de internações hospitalares por ano, verificou-se que dos 20 pacientes com este dado disponível, a metade (10 pacientes) apresentou ao menos uma hospitalização. Oito pacientes do grupo DC necessitaram de internação hospitalar, sendo que duas crianças necessitaram três e quatro internações por ano respectivamente, e os demais necessitaram, cada um, de uma internação. No grupo IRT dois pacientes necessitaram internação, sendo que cada um destes precisou de uma internação (Tabela 1).

Os 24 pacientes realizaram um total de 91 exames de espirometria (média de 3,8 exames por paciente). A idade da primeira espirometria foi de $4,46 \pm 0,74$ anos para os pacientes do grupo IRT e de $5,17 \pm 0,58$, para os do grupo DC (Tabela 1).

Foram encontrados valores discretamente superiores de $\mathrm{VEF}_{1}, \mathrm{CVF}$ e $\mathrm{FEF}_{25-75}$ nas espirometrias realizadas pelos pacientes do grupo IRT, embora estes não tenham atingido diferença estatisticamente significante para o tamanho amostral quando da comparação entre os grupos.

\section{DISCUSSÃO}

Não foi encontrado na literatura científica estudo semelhante, realizado no Brasil, que tenha avaliado o impacto da triagem neonatal para FC e, consequentemente, a possibilidade do seu diagnóstico mais precoce, na evolução das características clínicas, laboratoriais e espirométricas de pacientes em idade pré-escolar portadores dessa doença. Este estudo verificou um escore de Shwachman significativamente maior nas crianças do grupo IRT, em que o diagnóstico de FC havia sido feito a partir do TTN. Este achado é semelhante aos de estudos europeus $[6,16]$, mas contraria um estudo realizado no País de Gales no início da década de 90 [17]. O trabalho contribui, dessa forma, trazendo novas informações sobre o impacto da introdução do teste para FC no TTN em países em desenvolvimento.

O escore de Shwachman é uma ferramenta de avaliação clínico-radiológica, consagrada e utilizada não só nos serviços que acompanham pacientes com FC, mas também como medida de desfecho em pesquisa, como no estudo de Groves et al. [18]. Um dos quatro domínios abordados pelo escore é o status nutricional do paciente, refletindo a importância que este parâmetro possui na avaliação do indivíduo portador de FC. Embora não tenha sido estratificado o resultado de cada domínio do escore, pela característica de um estudo retrospectivo sem disponibilidade do dado original estratificado por domínios, é provável que o aspecto nutricional deva ter tido um impacto relevante no melhor escore obtido pelo grupo identificado por TTN. Um dado que colabora com esta hipótese é o resultado do IMC na comparação entre os grupos, cujo resultado teve significância estatística limítrofe a favor de uma melhor nutrição no grupo diagnosticado mais precocemente. Em um estudo realizado com pacientes australianos e norte-americanos, verificou-se melhor nutrição naqueles diagnosticados a partir da alteração do TTN [5]. Sabe-se que a desnutrição está associada a mais rápida progressão da doença pulmonar nos pacientes com FC, seja pelo comprometimento da musculatura da caixa torácica, pela diminuição da elasticidade pulmonar ou pelo prejuízo no sistema imunológico [19].

Estudos prévios que incluíram pacientes pediátricos de maior média de idade (abrangendo adolescentes) encontraram importante relação entre escore de Shwachman e testes de função pulmonar, especialmente $\mathrm{VEF}_{1}$ [20-22]. No presente estudo, embora verificada diferença nesse escore na comparação entre os grupos, não se observaram melhores parâmetros espirométricos no grupo IRT. Também não houve associação estatisticamente significativa entre IRT e função pulmonar. Pode-se considerar que a ausência dessas associações neste estudo seja devida ao pequeno 
tamanho da amostra ou ao curto período de tempo de observação (Tabela 1) [21].

Concordando com estudos prévios $[6,16,23]$, não se verificaram melhores resultados do $\mathrm{VEF}_{1}$ e da CVF entre os pacientes do grupo IRT em relação aos do grupo DC. Acredita-se que os motivos desse achado podem estar relacionados ao fato de a espirometria ser um método pouco sensível à obstrução das vias aéreas periféricas, ou, mais provavelmente, pelo pequeno tamanho amostral dos estudos que avaliaram essa associação. Além disso, embora crianças em idade préescolar consigam realizar manobras espirométricas, ainda apresentam dificuldade na execução se comparadas às mais velhas, mesmo aquelas com doença crônica [24]. Em função dessa observação, alguns estudos pediátricos, ao invés de considerarem o $\mathrm{VEF}_{1}$, recomendam o volume expiratório forçado na metade do primeiro segundo $\left(\mathrm{VEF}_{0,5}\right)$ ou a $75 \%$ do primeiro segundo $\left(\mathrm{VEF}_{0,75}\right)$ [24].

Neste estudo foi possível demonstrar diferença estatisticamente significativa entre as médias de idade em que os pacientes conseguiram realizar a primeira espirometria com sucesso, resultado que pode ser devido à precocidade tanto do acompanhamento ambulatorial quanto do estímulo à realização deste tipo de teste. Não se evidenciou diferença entre os grupos no que se refere à colonização por $P$. aeruginosa, em consonância com estudo prévio [6]. Uma hipótese para este resultado seria uma média de idade menor de nossa amostra comparada com a de outros estudos [7], onde houve um tempo maior para aquisição de $P$. aeruginosa mucoide, aquela correlacionada ao pior prognóstico da doença $[7,25]$.

Um dos principais fatores limitantes deste estudo foi o tamanho da amostra, restrita a 24 pacientes, além de ser um estudo retrospectivo de revisão de prontuários. É importante considerar que a recente inclusão do IRT no TTN universal no Rio Grande do Sul ainda determina um menor número de pacientes identificados pelo TTN. Por outro lado, deste momento em diante, a comparação entre esses grupos tende a ficar mais difícil, pois os pacientes diagnosticados por sinais e sintomas devem ser paulatinamente reduzidos a uma proporção muito baixa na população de pacientes com FC.

Os resultados do presente estudo indicam que os pacientes diagnosticados com FC a partir da suspeita pelo TTN beneficiaram-se de uma intervenção precoce, podendo iniciar mais cedo o seguimento com testes de função pulmonar e receber em tempo os necessários tratamentos e orientações. A avaliação de parâmetros clínicos com o escore de Shwachman mostrou que os benefícios puderam ser observados já na idade pré-escolar.

\section{NOTAS}

Apoio financeiro

Este estudo não recebeu apoio financeiro de fontes externas.

Declaração de conflito de interesses

Os autores declaram não haver conflitos de interesses relevantes ao conteúdo deste estudo.

Contribuições dos autores

Todos os autores fizeram contribuições substanciais para concepção, ou delineamento, ou aquisição, ou análise ou interpretação de dados; e redação do trabalho ou revisão crítica; e aprovação final da versão para publicação.

Disponibilidade dos dados e responsabilidade pelos resultados

Todos os autores declaram ter tido total acesso aos dados obtidos e assumem completa responsabilidade pela integridade destes resultados.

\section{REFERÊNCIAS}

1. Stocks J, Thia LP, Sonnappa S. Evaluation and use of childhood lung function tests in cystic fibrosis. Curr Opin Pulm Med. 2012;18(6):602-8. https://doi.org/10.1097/MCP.0b013e328358dfbe

2. Cantin AM, Hartl D, Konstan MW, Chmiel JF. Inflammation in cystic fibrosis lung disease: Pathogenesis and therapy. J Cyst Fibros. 2015;14(4):419-30. https://doi.org/10.1016/j.jcf.2015.03.003

3. Farrell PM, Rosenstein BJ, White TB, Accurso FJ, Castellani C, Cutting GR, Durie PR, Legrys VA, Massie J, Parad RB, Rock MJ, Campbell PW 3rd; Cystic Fibrosis Foundation. Guidelines for diagnosis of cystic fibrosis in newborns through older adults: Cystic Fibrosis Foundation consensus report. J Pediatr. 2008;153(2):S4-S14. https://doi.org/10.1016/j. jpeds.2008.05.005

4. Rosenfeld M. Overview of published evidence on outcomes with early diagnosis from large US observational studies. J Pediatr. 2005;147(3 Suppl):S11-4. https://doi.org/10.1016/j.jpeds.2005.08.010

5. Martin B, Schechter MS, Jaffe A, Cooper P, Bell SC, Ranganathan S. Comparison of the US and Australian cystic fibrosis registries: the impact of newborn screening. Pediatrics. 2012;129(2):e348-55. https://doi.org/10.1542/peds.2011-0567

6. Sims EJ, McCormick J, Mehta G, Mehta A; Steering Committee of the UK Cystic Fibrosis Database. Neonatal screening for cystic fibrosis is beneficial even in the context of modern treatment. J Pediatr. 2005;147(3 Suppl):S42-6. https://doi. org/10.1016/j.jpeds.2005.08.002 
7. Dijk FN, McKay K, Barzi F, Gaskin KJ, Fitzgerald DA. Improved survival in cystic fibrosis patients diagnosed by newborn screening compared to a historical cohort from the same centre. Arch Dis Child. 2011;96(12):1118-23. https:// doi.org/10.1136/archdischild-2011-300449

8. Hoo AF, Thia LP, Nguyen TT, Bush A, Chudleigh J, Lum S, Ahmed D, Balfour Lynn I, Carr SB, Chavasse RJ, Costeloe KL, Price J, Shankar A, Wallis C, Wyatt HA, Wade A, Stocks J; London Cystic Fibrosis Collaboration. Lung function is abnormal in 3-month-old infants with cystic fibrosis diagnosed by newborn screening. Thorax. 2012;67(10):874-81. https://doi.org/10.1136/thoraxjnl-2012-201747

9. Ranganathan S, Linnane B, Nolan G, Gangell C, Hall G. Early detection of lung disease in children with cystic fibrosis using lung function. Paediatr Respir Rev. 2008;9(3):160-7. https://doi.org/10.1016/j.prrv.2008.05.002

10. Shwachman H, Kulczycki LL. Long term study of one hundred five patients with cystic fibrosis. Am J Dis Child. 1958;96(1):6-15. https://doi.org/10.1001/archpedi.1958.02060060008002

11. Kozlowska WJ, Bush A, Wade A, Aurora P, Carr SB, Castle RA, Hoo AF, Lum S, Price J, Ranganathan S, Saunders C, Stanojevic S, Stroobant J, Wallis C, Stocks J; London Cystic Fibrosis Collaboration..Lung function from infancy to the preschool years after clinical diagnosis of cystic fibrosis. Am J Respir Crit Care Med. 2008;178(1):42-9. https://doi. org/10.1164/rccm.200710-15990C

12. Lee TW, Brownlee KG, Conway SP, Denton M, Littlewood JM. Evaluation of a new definition for chronic Pseudomonas aeruginosa infection in cystic fibrosis patients. J Cyst Fibros. 2003;2(1):29-34. https://doi.org/10.1016/S1569-1993(02)00141-8

13. Stanojevic S, Wade A, Cole TJ, Lum S, Custovic A, Silverman M, Hall GL, Welsh L, Kirkby J, Nystad W, Badier M, Davis S, Turner S, Piccioni P, Vilozni D, Eigen H, Vlachos-Mayer H, Zheng J, Tomalak W, Jones M, Hankinson JL, Stocks J;Asthma UK Spirometry Collaborative Group. Spirometry centile charts for young Caucasian children: the Asthma UK Collaborative Initiative. Am J Respir Crit Care Med. 2009;180(6):547-52. https://doi.org/10.1164/rccm.200903-0323OC

14. Heinzmann-Filho JP, Pinto LA, Marostica PJ, Donadio MV. Variation in lung function is associated with worse clinical outcomes in cystic fibrosis. J Bras Pneumol. 2015;41(6):509-15. https://doi.org/10.1590/s1806-37562015000000006

15. Miller MR, Hankinson J, Brusasco V, Burgos F, Casaburi R, Coates A, Crapo R, Enright P, van der Grinten CP, Gustafsson P, Jensen R, Johnson DC, MacIntyre N, McKay R, Navajas D, Pedersen OF, Pellegrino R, Viegi G, Wanger J; ATS/ERS Task Force. Standardisation of Spirometry. Eur Resp J. 2005;26(2):319-38. https://doi.org/10.1183/09031936.05.00034805

16. Siret D, Bretaudeau G, Branger B, Dabadie A, Dagorne M, David V, de Braekeleer M, Moisan-Petit V, Picherot G, Rault G, Storni V, Roussey M. Comparing the clinical evolution of cystic fibrosis screened neonatally to that of cystic fibrosis diagnosed from clinical symptoms: a 10-year retrospective study in a French region (Brittany). Pediatr Pulmonol. 2003;35(5):342-9. https://doi.org/10.1002/ppul.10259

17. Chatfield S, Owen G, Ryley HC, Williams J, Alfaham M, Goodchild MC, Weller P. Neonatal screening for cystic fibrosis in Wales and the West Midlands: clinical assessment after five years of screening. Arch Dis Child. 1991;66 (1 Spec No):29-33.

18. Groves T, Robinson P, Wiley V, Fitzgerald DA. Long-term outcomes of children with intermediate sweat chloride values in infancy. J Pediatr. 2015;166(6):1469-74. https://doi.org/10.1016/j.jpeds.2015.01.052

19. Castro LV, Martins MC. Application of Schwachman's scoring system to patients with cystic fibrosis with focus on the nutritional aspect. Rev Pediatr Ceara. 2005;6:27-36.

20. Stollar F, Adde FV, Cunha MT, Leone C, Rodrigues JC. Shwachman-Kulczycki score still useful to monitor cystic fibrosis severity. Clinics (Sao Paulo). 2011;66(6):979-83. https://doi.org/10.1590/S1807-59322011000600010

21. Assis I, Camargos PA, Reis FJ, Sulmonett N, Carneiro AP. Assessing correlations between spirometry and ShwachmanKulczycki score in children and adolescents. Pediatr Pulmonol. 2003;36(4):305-9. https://doi.org/10.1002/ppul.10350

22. Freire ID, Abreu E Silva FA, Araújo MA. Comparison among pulmonary function test results, the Shwachman-Kulczycki score and the Brasfield score in patients with cystic fibrosis. J Bras Pneumol. 2008;34(5):280-7. https://doi.org/10.1590/ S1806-37132008000500006

23. Farrell PM, Li Z, Kosorok MR, Laxova A, Green CG, Collins J, Lai HC, Rock MJ, Splaingard ML. Bronchopulmonary disease in children with cystic fibrosis after early or delayed diagnosis. Am J Respir Crit Care Med. 2003;168(9):1100-8. https://doi.org/10.1164/rccm.200303-434OC

24. Aurora P, Stocks J, Oliver C, Saunders C, Castle R, Chaziparasidis G, Bush A; London Cystic Fibrosis Collaboration. Quality control for spirometry in preschool children with and without lung disease. Am J Respir Crit Care Med. 2004;169(10):1152-9. https://doi.org/10.1164/rccm.200310-1453OC

25. Li Z, Kosorok MR, Farrell PM, Laxova A, West SE, Green CG, Collins J, Rock MJ, Splaingard ML. Longitudinal development of mucoid Pseudomonas aeruginosa infection and lung disease progression in children with cystic fibrosis. JAMA. 2005;293(5):581-8. https://doi.org/10.1001/jama.293.5.581 Є 